\title{
Bidirectional Light-Scattering Image Processing Method for High-Concentration Jet Sprays
}

\author{
ISAO SHIMIZU \\ Department of Mechanical Engineering, Ibaraki Technical College, Katsuta, Ibaraki, Japan
}

Y. EMORI

Department of Photographic Engineering, Chiba University, Chiba, Japan

WEN-JEI YANG

Department of Mechanical Engineering and Applied Mechanics, University of Michigan, Ann Arbor, MI 48109

and

M. SHIMODA and T. SUZUKI

Hino Motors, Hino, Tokyo, Japan

\begin{abstract}
In order to study the distributions of droplet size and volume density in high-concentration jet sprays, a new technique is developed, which combines the forward and backward light scattering method and an image processing method. A pulsed ruby laser is used as the light source. The Mie scattering theory is applied to the results obtained form image processing on the scattering photographs. The time history is obtained for the droplet size and volume density distributions, and the method is demonstrated by diesel fuel sprays under various injecting conditions. The validity of the technique is verified by a good agreement in the injected fuel volume distributions obtained by the present method and by injection rate measurements.
\end{abstract}

\section{INTRODUCTION}

The growth and diminution of liquid droplets in jet sprays, by condensation and evaporation, respectively, are of importance in many industrial systems and processes. For example, moisture induces efficiency losses and possibilities of erosion damage in steam turbines. In the combustion of liquid fuels, the evaporation rate of fuel droplets may be the rate-limiting step in the total process. Jet sprays are also applied in, among other things, the drying of viscous solutions by atomization, the spray cooling of hot gases and metals, and metal hardening or cutting.

The time history of both the droplet size and number density is essential in order to assess accurately the rate of evaporation or condensa- 
tion in jet sprays. Numerous methods have been employed for such purposes. For example, Kantola [1] determined the droplet size and number density time histories in steam condensation by three optical methods: attenuation, forward scattering, and side scattering techniques. These techniques were employed for small droplets $(0.1-1.5 \mu \mathrm{m})$. Trolinger [2] summarized particle field holography applications for larger droplets (over $5 \mu \mathrm{m}$ ). For applications in diesel engines, Shimizu [3] used a light scattering counter method to determine the dynamic behavior of low-concentration sprays. The holographic technique or light scattering counter method can be used to measure low-concentration sprays having number densities below 1000 particles $/ \mathrm{cm}^{3}$. In reality, the fuel is sprayed into a combustion chamber with a length of less than $100 \mathrm{~mm}$. In such cases, the spray is not greatly diffused and its density is thicker than 1000 particles $/ \mathrm{cm}^{3}$, while its droplet size is larger than $5 \mu \mathrm{m}$. Therefore, the techniques are not suitable for accurate measurements of the spatial distribution and timewise variation of the particle size and number density in dense sprays. For macroscopic measurement of a fuel spray with a relatively high concentration, high-speed photographic methods have been commonly employed. These techniques can continuously record the overall behavior of jet sprays, including diffusion and penetration depth. However, they cannot quantitatively evaluate the number density and particle size distributions. It is, therefore, desirable to develop a method for quantitative measurement of the spatial distributions of the droplet size and number density for jet sprays of relatively high concentration.

The present work combines the light attenuation theory, backward scattering theory, and image analysis to determine the time history of the spatial distributions of the droplet size and volume density along the entire passage of jet sprays. This technique is new and can be applied to fuel sprays of relatively high concentration to obtain high-quality results, by means of digital image processing of the photodensity on the light scattering photographs. The principle of the optical method and its application are presented.

\section{PRINCIPLE OF MEASUREMENTS}

When a single particle is situated in the parallel ray of a light beam of unit strength, the intensity of light scattering is given by the product of the Mie total-scattering coefficient $K_{\mathrm{s}}(\alpha)$ and the cross-sectional area of the particle. The particlesize parameter $\alpha$ is defined as $2 \pi r / \lambda$, where $r$ is the particle radius and $\lambda$ is the wavelength of the incident light. The light being scattered by a jet spray is split into backward and forward beams by a half-mirror $\mathrm{Hm} 1$ and lenses $\mathrm{L}_{3}$ and $\mathrm{L}_{5}$, as illustrated in Fig. 1. The scattered beams are then recorded by films $A$ and $B$ as will be explained in detail later. Let $I_{0}$ denote the incident light intensity; $n(\alpha)$, the number density; $l$, the spray thickness; $d x$, the differential length of the light passage; and $R_{\mathrm{t}}$ and $R_{\mathrm{b}}$, the collecting powers of the optical system for forward and backward scattering, respectively. The intensities of the forward and backward scattering are $[4,5]$

$$
\frac{I_{\mathrm{t}}}{I_{0}}=\exp \left[-\int_{0}^{l} \int_{0}^{\infty} R_{\mathrm{t}} K_{\mathrm{s}}(\alpha) \pi r^{2} n(\alpha) d \alpha d x\right]
$$

and

$$
\begin{aligned}
\frac{I_{\mathrm{b}}}{I_{0}} & =\int_{0}^{l} \int_{0}^{\infty} R_{\mathrm{b}} S(\alpha, \pi) n(\alpha) d \alpha \\
& \times\left\{\exp \left[-\int_{0}^{x} \int_{0}^{\infty} K_{\mathrm{s}}(\alpha) \pi r^{2} n(\alpha) d \alpha d x\right]\right\}^{2} d x,
\end{aligned}
$$

respectively. Here, $S(\alpha, \pi)$ represents the backward scattering function [5, 6]. Figure 2 depicts a schematic diagram of the forward and backward scattering of light through a particle field.

Let us define the extinction coefficient $\xi$ and the ratio of reflectivity to extinction $\zeta$ as

$\xi=\int_{0}^{\infty} K_{\mathrm{s}}(\alpha) \pi r^{2} n(\alpha) d \alpha$ 


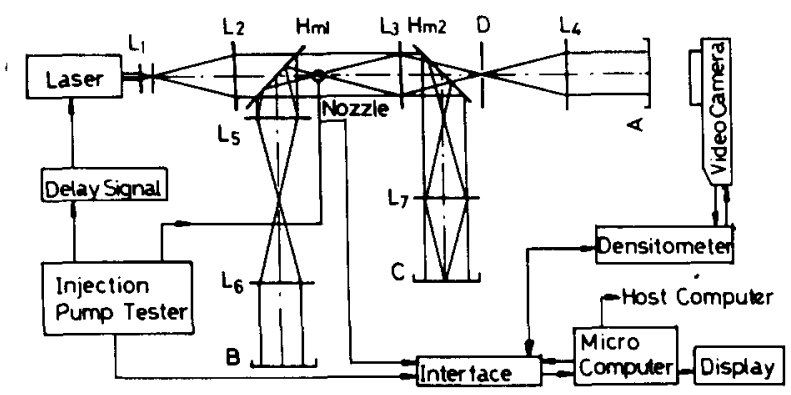

Fig. 1. A schematic diagram of optical system and test apparatus for jet spray photography.

and

$$
\begin{aligned}
\zeta= & \int_{0}^{\infty} S(\alpha, \pi) n(\alpha) d \alpha \\
& \times / \int_{0}^{\infty} K_{\mathrm{s}}(\alpha) \pi r^{2} n(\alpha) d \alpha .
\end{aligned}
$$

In general, $R_{\mathrm{t}}$ and $R_{\mathrm{b}}$ for each particle vary with location on the light passage due to a change in the scattering position of the particle. However, when particle clouds of a jet spray are viewed from a distance, $R_{\mathrm{t}}$ and $R_{\mathrm{b}}$ of each particle may be considered constant. Then, Eqs. (1)-(4) can be combined to become

$$
\begin{aligned}
& \frac{I_{\mathrm{t}}}{I_{0}}=R_{\mathrm{t}} \exp \left(-\int_{0}^{l} \xi d x\right) \\
& \frac{I_{\mathrm{t}}}{I_{0}}=R_{\mathrm{b}} \int_{0}^{l} \zeta \xi\left[\exp \left(-2 \int_{0}^{x} \xi d x^{\prime}\right)\right] d x
\end{aligned}
$$

(through an integration by parts)

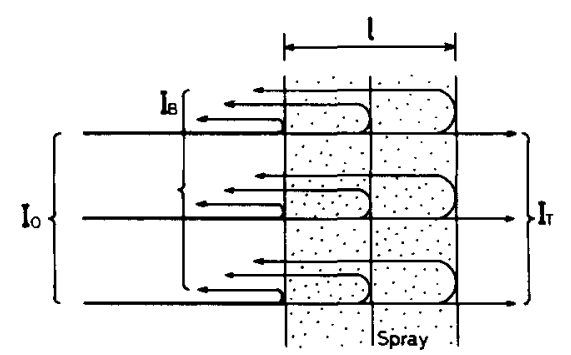

Fig. 2. Backward scattering and transmission of light.

$$
\begin{aligned}
= & \frac{R_{\mathrm{b}}}{2}\left\{\left[\zeta_{0}-\zeta_{l} \exp \left(-2 \int_{0}^{l} \xi d x\right)\right]\right. \\
& \left.+\int_{0}^{l} \frac{d \zeta}{d x} \exp \left(-2 \int_{0}^{x} \xi d x^{\prime}\right) d x\right\} .
\end{aligned}
$$

Here, $\zeta_{0}$ and $\zeta_{l}$ denote the values of $\zeta$ at $x=0$ and $x=l$, respectively. $\xi$ is a function of $x$ because it is generally dependent upon the particle size and number density distributions for the light passage. The effect of multiple scattering is assumed negligible in Eqs. (5) and (6), even for very dense sprays, as in the initial stage of diesel sprays, so long as the receiver lens aperture is very small in the measurement of large particles of over $5-\mu \mathrm{m}$ diameter $[6,11]$.

Both $I_{\mathrm{t}}$ and $I_{\mathrm{b}}$ must be determined experimentally. Then, Eqs. (5) and (6) can be used to evaluate one of the three parameters $l, r$ and $n(\alpha)$, provided the other two are known. Twomey and Howell [5] evaluated the values of $\zeta$ for a monochromatic wave of $\lambda=0.7 \mu \mathrm{m}$ and a white light with $\lambda=0.4-0.7 \mu \mathrm{m}$ assuming that $n(\alpha)$ in Eq. (4) takes the Gaussian, Poisson, bimodal, or inverse Poisson distribution. Shimizu et al. [6] found that for particles of a diameter between 1 and $60 \mu \mathrm{m}$, the values of $\zeta$ for the monochromatic wave fluctuate rigorously about the value of $\alpha$, while $\zeta$ for the white light takes a value practically proportional to the particle diameter. Shimizu et al. [6] measured $I_{\mathrm{b}}$ for monochromatic waves and observed a proportionality between $\zeta$ and the mean particle radius $r_{10}$,

$\zeta=a r_{10}$, 
where $a$ is the proportionality constant and

$r_{10}=\int_{0}^{\infty} r n(\alpha) d \alpha / \int_{0}^{\infty} n(\alpha) d \alpha$

For homogeneous particles, $d \zeta / d x=0$ and $\zeta_{l}$ $=\zeta_{0}$ in Eq. (6), which can be reduced to

$I_{\mathrm{b}} / I_{0}=\frac{R_{\mathrm{b}}}{2}\left\{\zeta_{0}\left[1-\exp \left(-2 \int_{0}^{l} \xi d x\right)\right]\right\}$.

In the case of homogeneous and heterogeneous particles with droplet and density symmetrically distributed in the light beam direction $[7,8]$, Eq. (6) with the above equation can be reduced to

$\frac{I_{\mathrm{b}}}{I_{0}}=\frac{R_{\mathrm{b}}}{2}\left\{\bar{\zeta}\left[1-\exp \left(-2 \int_{0}^{l} \xi(x) d x\right)\right]\right\}$,

in which $\bar{\zeta}$ represents the average value of $\zeta(x)$ along the light passage. For heterogeneous particles [7], the value of $I_{\mathrm{b}} / I_{0}$ obtained using Eq. (9) differs from that using Eq. (6) by less than $10 \%$. This difference is within $20 \%$ even for jet sprays with higher number density and narrower spray width.

Equations (5) and (9) are combined to eliminate the $\int_{0} \xi d x$ term, resulting in

$\bar{\xi}=\left(\frac{2 R_{\mathrm{t}}^{2}}{R_{\mathrm{b}}}\right)\left(\frac{I_{\mathrm{b}}}{I_{0}}\right) /\left[R_{\mathrm{t}}^{2}-\left(\frac{I_{\mathrm{t}}}{I_{0}}\right)^{2}\right]$.

The value of $R_{\mathrm{t}}$ is unity if the parallel incident beam after passing through the spray is completely intercepted by the collecting lens. With the aid of Eqs. (7) and (10), one can determine the mean particle radius as

$\bar{r}_{10}=A_{1} \frac{\left(I_{\mathrm{b}} / I_{0}\right)}{\left[1-\left(I_{\mathrm{t}} / I_{0}\right)^{2}\right]}$,

in which $A_{1}$ is a constant that must be empirically determined.

Reference [5] indicated that for $\alpha \geqq 30$, the value of $K_{\mathrm{s}}(\alpha)$ is almost constant at $2[9,10]$. $\xi$ defined by Eq. (3) can, then, be rearranged as

$\xi=\pi K_{\mathrm{s}} r_{20} N$, in which

$r_{20}=\int_{0}^{\infty} r^{2} n(\alpha) d \alpha / N, \quad N=\int_{0}^{\infty} n(\alpha) d \alpha$.

$N$ signifies the number of particles of all sizes per unit volume. The volume density $M$ is defined as

$M=\int_{0}^{\infty} r^{3} n(\alpha) d \alpha$

It can be rearranged to read

$M=r_{32} r_{20} N$,

in which

$r_{32}=\int_{0}^{\infty} r^{3} n(\alpha) d \alpha / \int_{0}^{\infty} r^{2} n(\alpha) d \alpha$.

By using $r_{10} \cong r_{32} / 1.36$ [6], Eqs. (7), (12), and (15) are combined to yield

$M \propto \zeta \xi$.

Combining this relationship with the definitions

$\bar{\zeta}=\int_{0}^{x} \zeta d x / x, \quad \xi=\int_{0}^{x} \xi d x / x$

together with Eqs. (5) and (9), one obtains

$\bar{M}=A_{2} \frac{I_{\mathrm{b}} / I_{0}}{\left[1-\left(I_{\mathrm{t}} / I_{0}\right)^{2}\right]} \frac{\left[-\log \left(I_{\mathrm{t}} / I_{0}\right)\right]}{l}$,

in which $A_{2}$ is a proportionality constant.

The photographic density $F_{\mathrm{D}}$ is related to the film exposure $E$ by

$F_{\mathrm{D}}=\gamma \log E=\gamma \log (I t)$.

Here, $\gamma$ denotes the gamma value of film and $E$ is the product of the intensity of light $I$ and the exposure time $t$. Let the films be exposed over a unit time by the forward and backward scattering lights, i.e., $t=1$ time unit. Then, the substitutions of Eq. (19) into Eqs. (11) and (18) yield $\bar{r}$ and $\bar{M}$. 


\section{EXPERIMENTAL APPARATUS AND PROCEDURE}

Figure 1 is a schematic diagram of an optical recording system and a digital processing unit. The film A recorded the extinct light as a forward photography, while the film B recorded a backward scattering photograph. $\mathrm{C}$ was an optical system for recording either a laser hologram or a schlieren photograph. $\mathrm{L}_{2}-\mathrm{L}_{5}$ were all $60 \mathrm{~mm}$ diameter lenses of $200 \mathrm{~mm}$ focal length. In order to approximate the total scattering coefficient $K_{\mathrm{s}}(\alpha)$ as a constant, a diaphragm $D$ was placed at the focal point of the lens $L_{3}$ to receive only the parallel scattering light [10].

A pulsed laser of $2 \mathrm{~J}$ output and $2 \mathrm{~ns}$ flash time was used as the light source. Any delay time may be set between the start of fuel injection and the activation of the pulsed laser. A Kodak photographic step tablet (or gray scale) was recorded on the film together with the jet spray. The gray scale was then used as the reference to detemine the photographic density and the gamma value of the film. $35 \mathrm{~mm}$ Kodak Tri X Pan, Recording 2475, and Agfa 10E75 films were used in the study. A Hamamatsu C-1000 type video camera, an NAC 1200 type B color density meter, and a microcomputer controlled image processing unit were employed for image analysis. A diesel fuel (No. 2 kerosene) was sprayed horizontally into an open atmosphere through a $0.39 \mathrm{~mm}$ diameter nozzle. The injection pump was a Bosch P-type with a $12 \mathrm{~mm}$ diameter plunger. Tests were conducted at two pump speeds: 500 and $1200 \mathrm{rpm}$. The fuel injection rates were $120.0 \mathrm{~mm}^{3} /$ stroke at 500 $\mathrm{rpm}$ and $135.0 \mathrm{~mm}^{3} / \mathrm{stroke}$ at $1200 \mathrm{rpm}$. The opening pressure of the nozzle was $21.6 \mathrm{MPa}$ or $220 \mathrm{Kgf}$. The injection pump speed, injection timing, and duration time were varied. Figure 3 depicts the timewise variations of the injection rate and pressure. The elapsed time was measured from the instant the fuel was ejected from the nozzle. By using the data in Fig. 3, the injection rate-to-pressure ratio was found to be approximately constant after $100 \mu \mathrm{s}$, resulting in a direct proportionality relationship between the injection rate and pressure.

Photographs of each jet spray were taken every $100 \mu$ s after the fuel injection was initiated. A typical set of forward and backward scattering photographs for $500 \mathrm{rpm}$ of injection pump speed are illustrated in Figs. 4(a) and 4(b).

\section{IMAGE PROCESSING}

An NAC 1200 type B color densitometer was used to determine photographic density of forward and backward scattering photographs of Fig. 4 and to express the density difference by shade as depicted in Fig. 5(a) and (b) for forward and backward scattering, respectively. The density distributions exhibited in the graphics in Fig. 5(a) and (b) were read digitally and then diagramatically illustrated in Fig. 6. These instantaneous spatial distributions of $I_{\mathrm{b}} / I_{0}$ and $I_{\mathrm{b}} /$ $I_{0}$ corresponded to $600 \mu \mathrm{s}$ after the initiation of fuel injection for a pump speed of $500 \mathrm{rpm}$. The spray width $l$ was directly measured from the

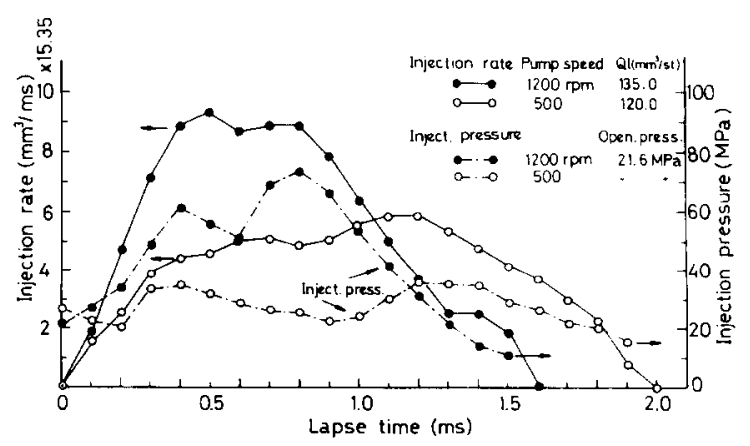

Fig. 3. Time history of the injected rate and pressure. 

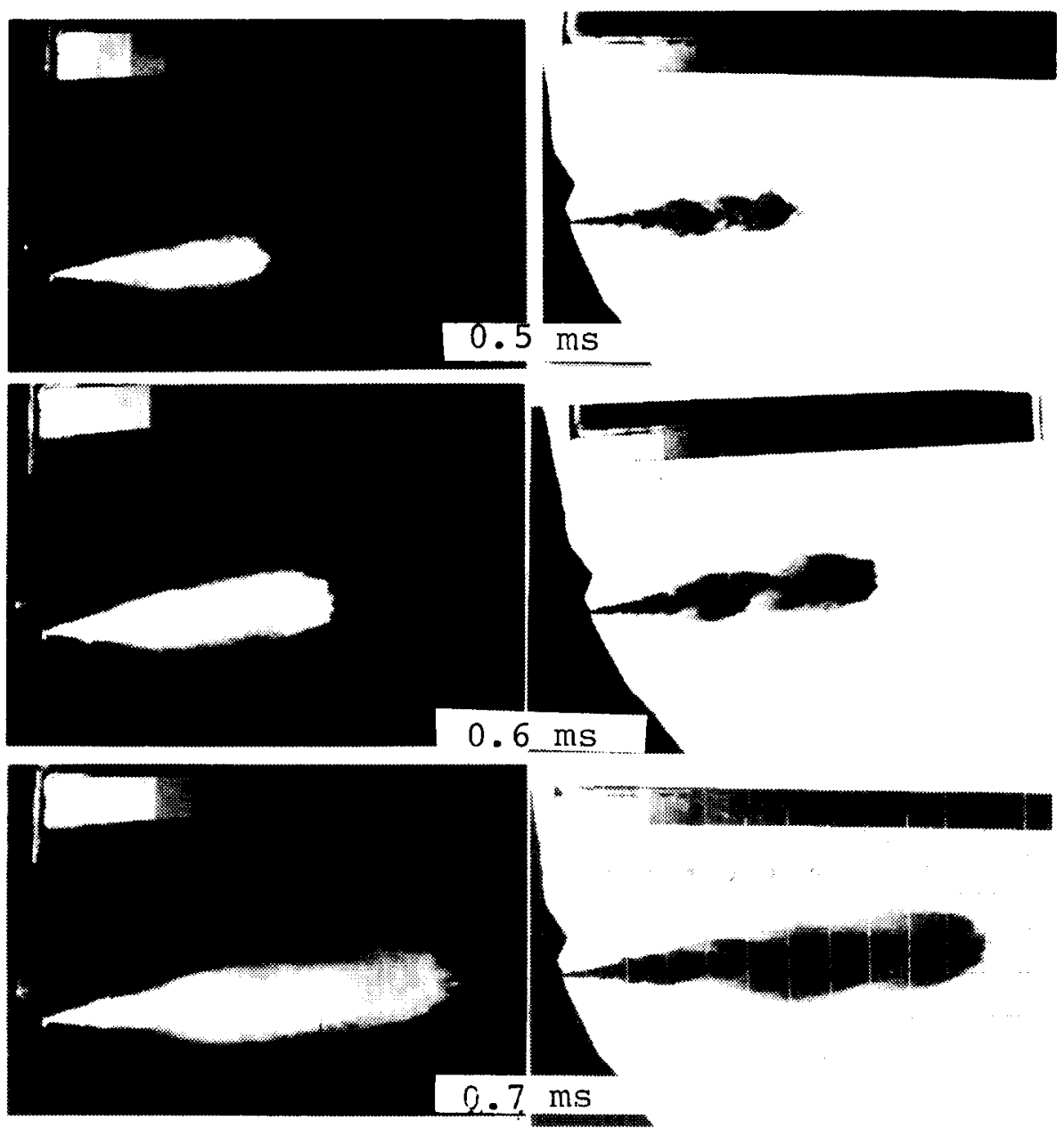

(a)

(b)

Fig. 4. Images of (a) backward and (b) forward scattering of a jet spray at $500 \mathrm{rpm}$ pump speed. 

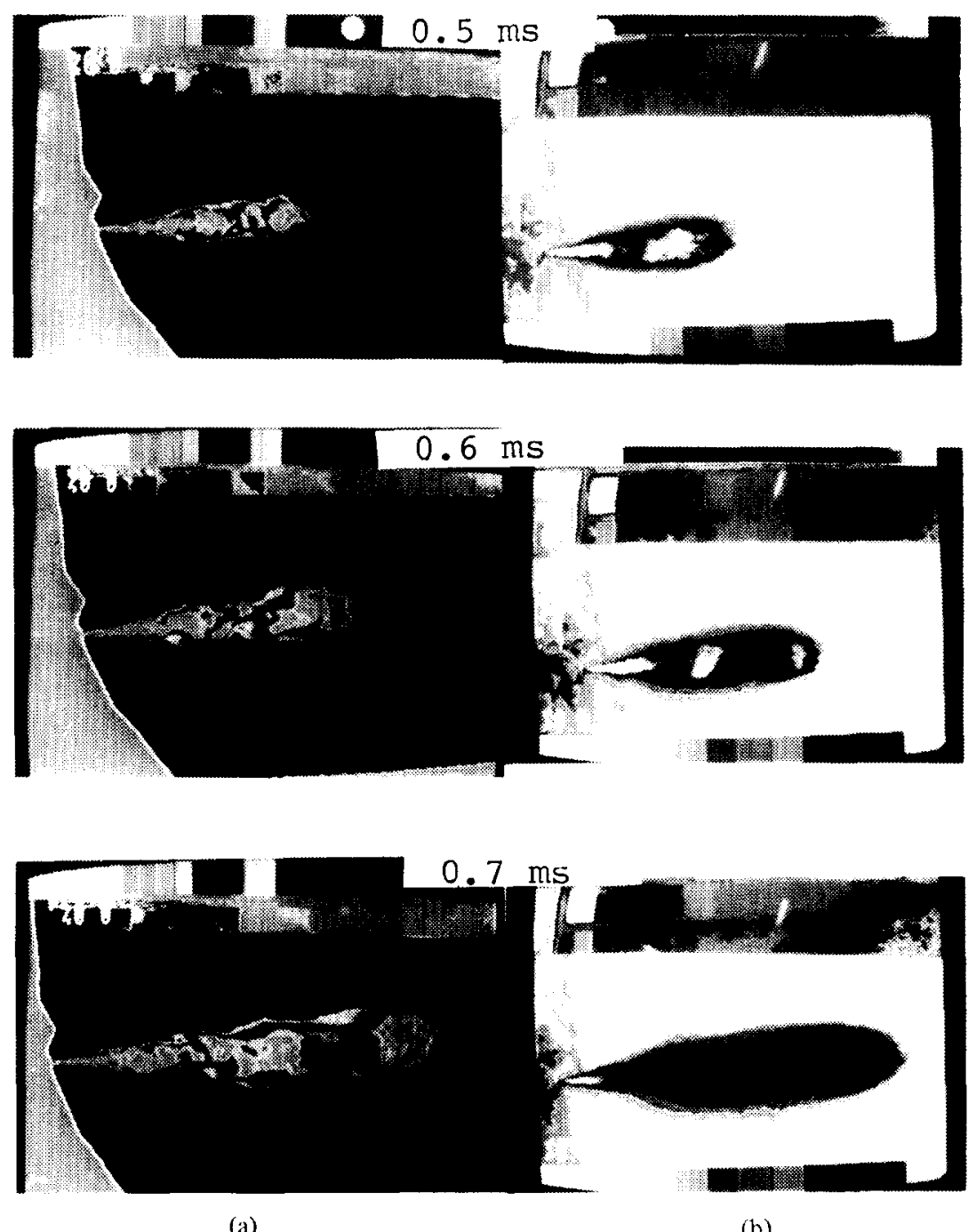

(a)

(b)

Fig 5. Representation of image concentrations of (a) forward and (b) backward scattering of a jet spray at $500 \mathrm{rpm}$ pump speed. 


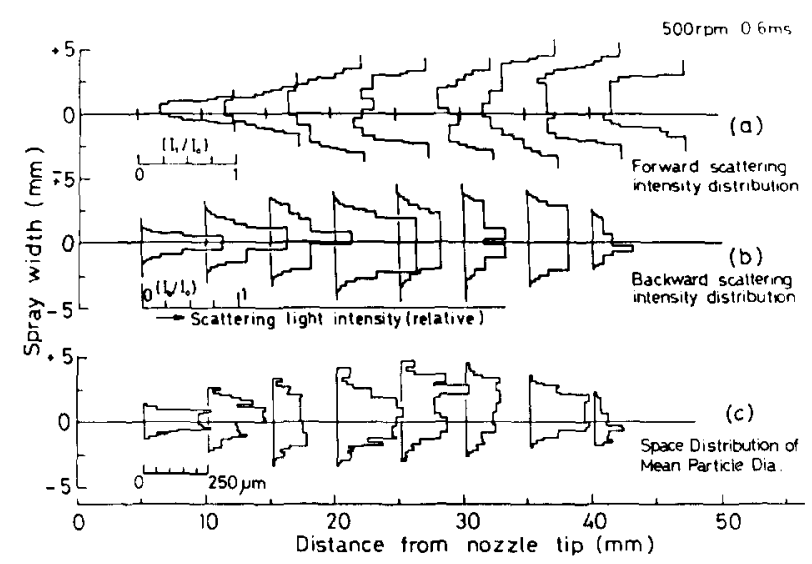

Fig. 6. Spatial distributions of (a) forward scattering, (b) backward scattering, and (c) mean particle diameter of a jet spray at $0.6 \mathrm{~ms}$ and $500 \mathrm{rpm}$ pump speed.

photograph (Fig. 4). Each segment manifests the result at a location of the vertical baseline. The vertical dimension measures the spray width, while the horizontal length from the baseline represents the magnitude of the physical quantity, $I_{\mathrm{t}} / I_{0}$ (with the baseline at right), $I_{\mathrm{b}} /$ $I_{0}$, mean droplet diameter, or volume density of the jet spray. Its instantaneous spatial distribution at $0.6 \mathrm{~ms}$ and $500 \mathrm{rpm}$ is illustrated in Fig. $7 \mathrm{a}$ assuming the spray to be symmetrical with the light beam. By using the intensity distributions in Figs. $6 \mathrm{a}$ and $6 \mathrm{~b}$ as $I_{\mathrm{t}} / I_{0}$ and $I_{\mathrm{b}} / I_{0}$, respectively, Eq. (11) with an arbitrary value of $A_{1}$ determined the mean particle radius $\bar{r}_{10}$. The instantaneous spatial distribution of the mean particle diameter is depicted in Fig. 6c. By using $\bar{r}_{10}$ in Fig. $6 \mathrm{c}$ and $I_{\mathrm{t}} / I_{0}$ from Fig. 6a together with an arbitrary value of $A_{2}, \mathrm{Eq}$. (18) determined the volume density of the spray. Figure $7 a$ depicts the cross-sectional view of the mean diameter distribution, while Fig. 7(b) shows the instantaneous spatial distribution of volume density at $0.6 \mathrm{~ms}$ and $500 \mathrm{rpm}$. It is important to note that the values of $\bar{r}_{10}$ and $\bar{M}$ were evaluated using the arbitrarily selected values of $A_{1}$ and $A_{2}$.

\section{v. CALIBRATIONS}

Holography was employed in the present study to determined the particle size. It has sensitivity for droplets larger than $5 \mu \mathrm{m}$ in diameter. The contribution of small droplets in the spray was neglected since diesel sprays are known to contain less droplets of diameters smaller than 5 $\mu \mathrm{m}[7,8,13]$. The constants $A_{1}$ and $A_{2}$ must be

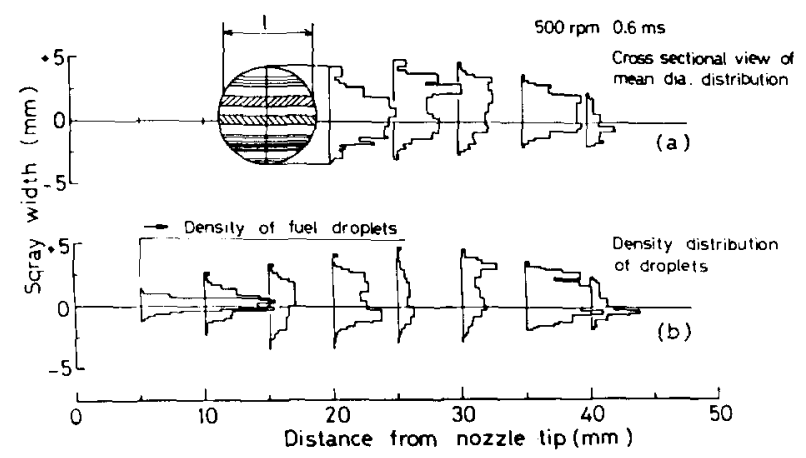

Fig. 7. Spatial distributions of (a) mean particle diameter and (b) density at $0.6 \mathrm{~ms}$ and 500 rpm pump speed. 


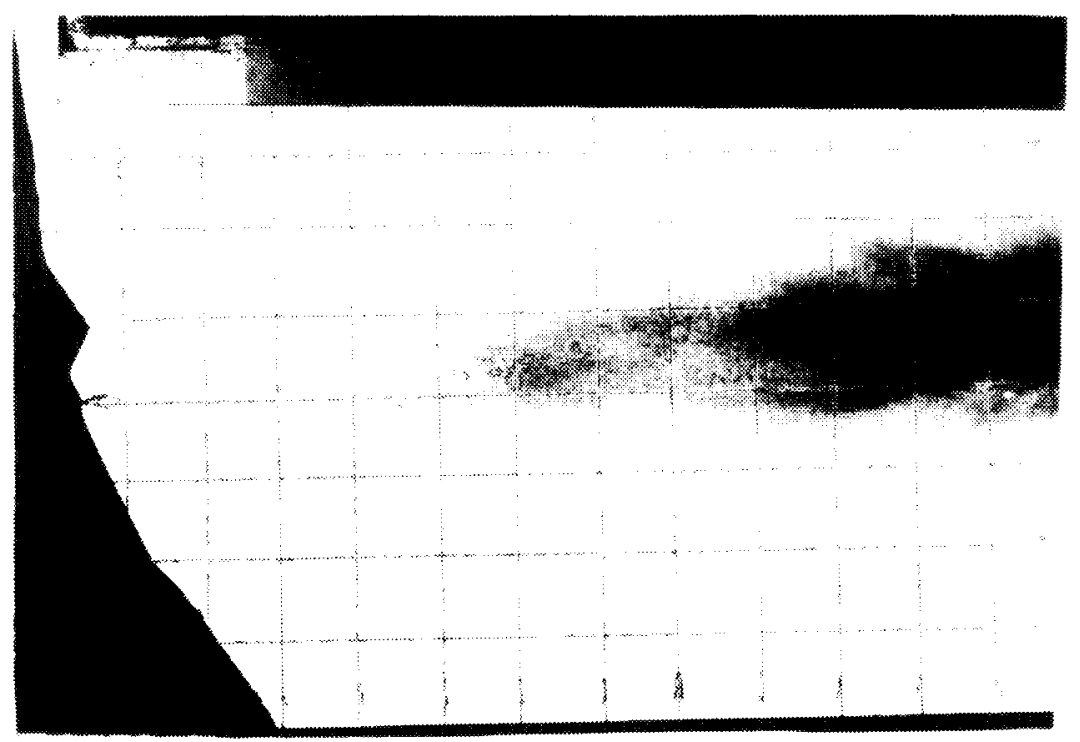

Fig. 8. Hologram of a jet spray at $1.8 \mathrm{~ms}$ and $1200 \mathrm{rpm}$ pump speed.

determined in order to calculate correct values of the mean particle radius and the volume density using Eqs. (11) and (18), respectively. An in-line hologram was taken at location $\mathrm{C}$ in Fig. 1, simultaneously with the forward and backward scattering photographs. Figure 8 depicts the hologram of a jet spray at $1.8 \mathrm{~ms}$ after the initiation of fuel injection using a fuel pump at a speed of $1200 \mathrm{rpm}$. Each side of the square grid in the hologram is about $5 \mathrm{~mm}$ in length. The constant $A_{1}$ in Eq. (11) was determined by equating $\bar{r}_{10}$ measured from the reconstruction of the droplet image on the hologram to $\bar{r}_{10}$ determined by the image processing of the corresponding scattering photographs.
Figure 9 depicts an instantaneous spatial distribution of the actual mean droplet diameter determined by Eq. (11), at the instant of $1.8 \mathrm{~ms}$ and $1200 \mathrm{rpm}$ of fuel pump speed. The results indicate that the mean droplet diameters are approximately uniform at each location along the flow path.

The constant $A_{2}$ was evaluated by equating the total volume of injected liquid obtained from the measured injection rates over the entire injection time to the total amount of liquid injection obtained by integrating the volume density distribution over the entire spray penetration (which was obtained from the image processing of the scattering photograph). The

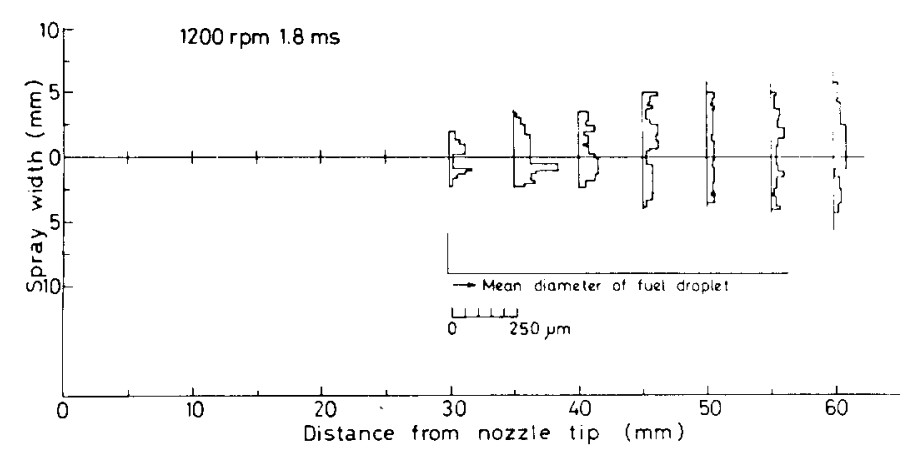

Fig. 9. Spatial distributions of mean particle diameter at $1.8 \mathrm{~ms}$ and $1200 \mathrm{rpm}$ pump speed. 


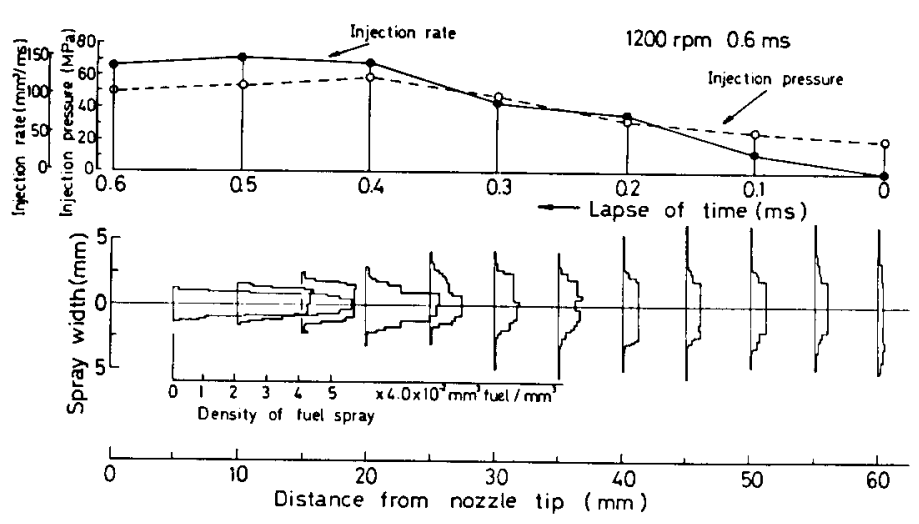

Fig. 10. Spatial distribution of volume density at $0.6 \mathrm{~ms}$ and time history of the injection rate and pressure at $1200 \mathrm{rpm}$ pump speed.

value of $A_{2}$ varies with the pump speed and elapsed time: $A_{2}=0.764,0.785$, and 0.811 at $0.6,0.7$, and $0.8 \mathrm{~ms}$, respectively, for the pump speed of $500 \mathrm{rpm}$, while $A_{2}=0.686$ and 0.666 at 0.4 and $0.5 \mathrm{~ms}$, respectively, for $1200 \mathrm{rpm}$. These $A_{2}$ values under various operating conditions differ less than about $20 \%$. The small deviation is evidence that this method meets the requirement for precise measurements of high density sprays.

Equation (18) was employed to calculate the actual instantaneous spatial distributions of the volume density at $0.6,1.2$, and $1.8 \mathrm{~ms}$ for the pump speed of $1200 \mathrm{rpm}$ using $A_{2}=0.764$.
Results are shown in Figs. 10-12, together with the time histories of the injection rate and pressure. Notice that the time scale (increasing from right to left) is opposite to the direction of the jet spray. The density segments were plotted at $5 \mathrm{~mm}$ increments along the spray path. The time interval used equals the path increment divided by the average spray velocity, which was empirically determined from spray photographs to be $100 \mathrm{~m} / \mathrm{s}$. It is observed that as the time elapses from 0 to $0.4 \mathrm{~ms}$, both the injection pressure and rate increase, while the volume density is higher in the region close to the nozzle opening. The injection rate remains fairly con-
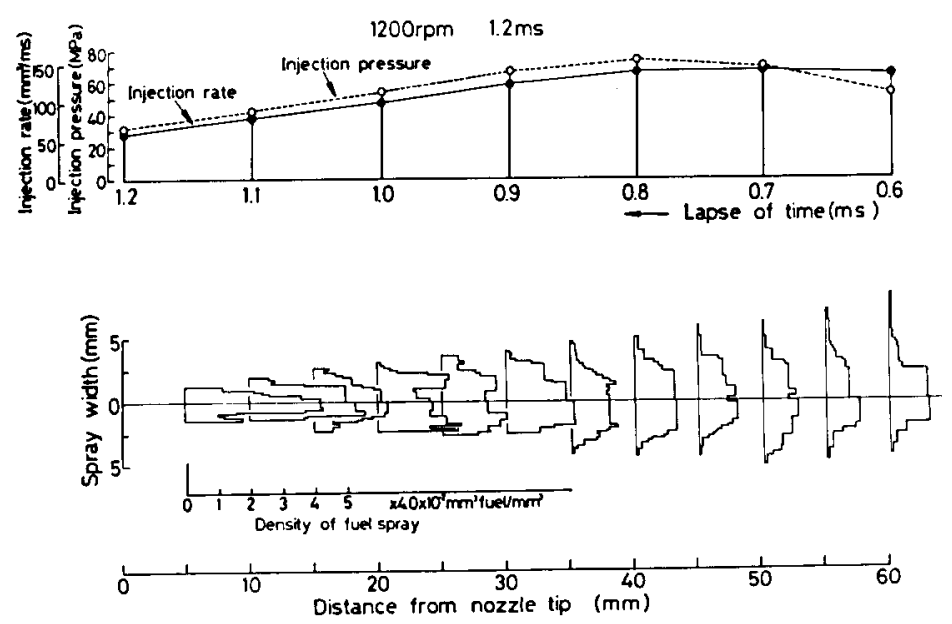

Fig. 11. Spatial distribution of volume density at $1.2 \mathrm{~ms}$ and time history of the injection rate and pressure at $1200 \mathrm{rpm}$ pump speed. 

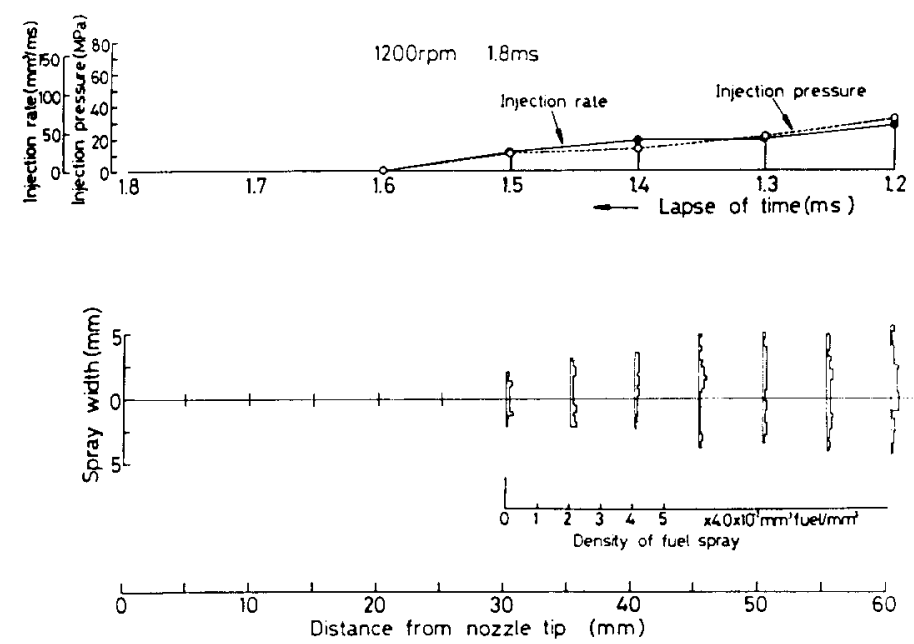

Fig. 12. Spatial distribution of volume density at $1.8 \mathrm{~ms}$ and time history of the injection rate and pressure at $1200 \mathrm{rpm}$ pump speed.

stant from 0.4 to $0.8 \mathrm{~ms}$, then followed by a gradual decrease to zero at $1.6 \mathrm{~ms}$. The injection pressure reaches a maximum at $0.8 \mathrm{~ms}$ and diminishes thereafter. The injected fuel volume distribution calculated by the volume density distribution agrees well with that calculated by the injected rate measurement, as shown in Fig. 13. The validity of the image density method in determining the volume density distribution is verified by this comparison and by the fact that deviations in the values of $A_{2}$ are less than $10 \%$ under various operating conditions.

\section{CONCLUSIONS}

In order to determine quantitatively the spatial distributions of the mean droplet diameter and volume density in high concentration jet sprays, a method is developed which processes the images of forward and backward scattering photographs of the spray. It is concluded that

i. the combined forward and backward light scattering method is suitable for measuring the droplet size and volume density of a high concentration jet spray,

ii. the image density method can be employed to determine the time histories of distributions of the droplet size and volume density,

iii. the method is unique in its applicability to high-concentration jet sprays which cannot be handled by conventional light scattering and holographic methods,

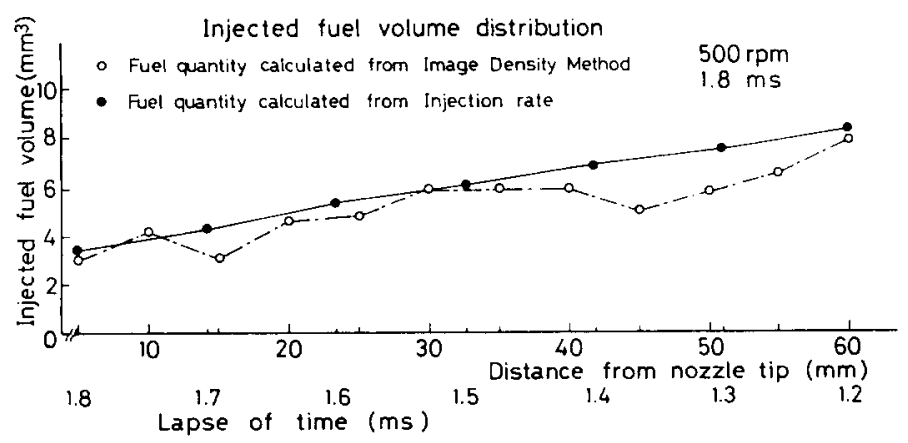

Fig. 13. Comparison of injected fuel volume distribution calculated by volume density distribution by injected rate measurement. 
iv. the validity of the new technique is confirmed by good agreement between the injected fuel volume distributions obtained by the present image processing method and those determined by injection rate measurements, and

$v$. the present study demonstrates its application to diesel fuel sprays.

\section{REFERENCES}

1. Kantola, R. A., in Heat and Mass Transfer in Rotating Machinery (Metzger, Ed.) Hemisphere, Washington, D.C., 1983.

2. Trolinger, J. D., and Field, D., AIAA Paper No. 800018, presented at the AIAA 18th Aerospace Science Meeting, Jan. 14-16, 1980, Pasadena.
3. Shimizu, I., Trans. Japan Soc. Mech. Engrs., Ser. B 47:2369-2378 (1981).

4. Gumprecht, K. O., and Sliepcevich, C. M., J. Phys. Chem. 57:90-95 (1953).

5. Twomey, S., and Howell, H. B., Appl. Optics 4:501-506 (1965).

6. Shimizu, H., Kobayashi, T., and Inaba, H., J. Appl. Phys. (Japanese) 47:30-36 (1978).

7. Itoh, I., Trans. Japan Soc. Mech. Engrs., Ser. B 36:759-780 (1970).

8. Tanabe, H., Yamamoto, H., Takahashi, Y., Kuniyoshi, H., Fujimoto, H., and Satoh, G., Japan Soc. Mech. Engrs. Preprint No. 821-3, 1982, pp. 281 287.

9. Sinclair, D., J. Opt. Soc. Am. 37:475-480 (1947).

10. Lothian. G. F., and Chappel, F. P., J. Appl. Chem. 1:475-482 (1951).

Received 9, January 1984; revised 13 August 1984 